\title{
ESTROGÊNIOS EM ÁGUAS NATURAIS E TRATADAS DA REGIÃO DE JABOTICABAL - SÃO PAULO
}

Laudicéia G. Lopes*

Serviço Autônomo de Água e Esgoto de Jaboticabal, Av. Jornalista Cláudio Luís Berchielli, 345, 14871-570 Jaboticabal - SP, Brasil Mary R. R. Marchi, João B. G. Souza e José A. Moura

Departamento de Química Analítica, Instituto de Química, Universidade Estadual Paulista, R. Francisco Degni, s/n, 14800-900 Araraquara - SP, Brasil

Cintia S. Lorenzon, Claudinei Cruz e Luiz A. Amaral

Faculdade de Ciências Agrárias de Veterinárias, Universidade Estadual Paulista, Via de Acesso Prof. Paulo Donato Castellane, s/n, 14884-900 Jaboticabal - SP, Brasil

Recebido em 5/5/09; aceito em 14/9/09; publicado na web em 12/2/10

ESTROGENS IN NATURAL AND TREATED WATERS IN THE REGION OF JABOTICABAL - SÃO PAULO, BRAZIL. The aim of this work was to analyze $17 \beta$ estradiol and estrone, natural estrogenic hormones present in domestic effluents and animal excreta, in the public water supply system of Jaboticabal, SP. The results have shown the presence of estrogens in $22 \%$ of the samples in concentrations from $6,8 \mathrm{ng} \mathrm{L}^{-1}$ (treated water) to $30,6 \mathrm{ng} \mathrm{L}^{-1}$ (riverhead) for $17 \beta$ estradiol and $600 \mathrm{ng} \mathrm{L}^{-1}$ of estrone (stream), respectively. We concluded that animal wastes, discharges from the wastewater treatment station, and discharges of domestic effluent without treatment from rural proprieties were probably the cause of this contamination.

Keywords: endocrine disruptor; potable water; watershed.

\section{INTRODUÇÃO}

A presença de micropoluentes em água - poluentes presentes em concentrações de $\mu \mathrm{g} \mathrm{L}^{-1}$ e ng L $\mathrm{L}^{-1}$ - tem despertado o interesse da comunidade científica. Fármacos, interferentes endócrinos e poluentes orgânicos persistentes (POP) são as classes mais investigadas pelos efeitos no meio ambiente. ${ }^{1}$ Os interferentes endócrinos são definidos como agentes exógenos que interferem na síntese, transporte, recepção, ação, ou eliminação dos estrogênios naturais do corpo e abrangem uma grande faixa de classes de estruturas distintas, dentre as quais estão os hormônios estrogênicos ou estrogênios. ${ }^{2}$

Os estrogênios são produzidos naturalmente pelos organismos ou sintetizados industrialmente para serem utilizados como fármacos. ${ }^{3}$ Um dos primeiros estudos correlacionando a dispersão ambiental de estrogênios naturalmente excretados por humanos e efeitos ecotoxicológicos foi realizado na Inglaterra, com peixes, a jusante de pontos de lançamento de estações de tratamento de esgoto (ETEs). Os peixes machos produziam a proteína vitelogenina, normalmente produzida por fêmeas sob a influência do hormônio estrogênico $17 \beta$ estradiol. ${ }^{4}$ Além da feminilização de peixes machos, ${ }^{5}$ a exposição prolongada aos estrogênios também tem sido associada com o aumento da incidência de câncer de mama, ${ }^{6}$ de testículos e da infertilidade masculina. ${ }^{7}$

A dispersão no ambiente dá-se após serem eliminados pelo esgoto, onde ocorre o lançamento direto nos corpos d'água ou após a passagem por tratamento. ${ }^{8} \mathrm{~A}$ destinação no solo também é possível pela deposição de resíduos animais, que posteriormente atingem mananciais superficiais por escoamento, ou aquíferos por infiltração. ${ }^{8}$

Os estrogênios naturais (estrona, $17 \beta$ estradiol e estriol) foram encontrados no esgoto in natura, ${ }^{9-12}$ no esgoto tratado, ${ }^{9-12}$ em água superficial ${ }^{13-17}$ e em água tratada. ${ }^{14-16} \mathrm{O}$ estrogênio sintético etinilestradiol, utilizado para contracepção, também está presente no ambiente, no entanto foi encontrado em menores concentrações que os naturais. ${ }^{10,11,13-15,17-19}$

A excreção diária de estrogênios pela mulher é de 106, 14 e 32 $\mu \mathrm{g}$ de estriol, $17 \beta$ estradiol e estrona, respectivamente. Esses se en-

*e-mail: laudiceia_lopes@globo.com contram inativos, conjugados ao ácido glucurônico (17 $\beta$ estradiol) ou ao sulfato (estrona). ${ }^{20} \mathrm{O}$ rompimento da conjugação ocorre no contato com a população de coliformes termotolerantes, produtora das enzimas glucuronidase e arilsulfatase,${ }^{20}$ tornando os estrogênios lançados nos corpos d'água biologicamente ativos. A atividade dos estrogênios pode ser minimizada por mecanismos de biodegradação, ${ }^{21}$ de sorção aos sedimentos suspensos ${ }^{22}$ e aos sedimentos do leito do corpo d'água, ${ }^{23}$ adsorção ao solo ${ }^{24}$ e fotólise..$^{25}$

As águas captadas em mananciais superficiais podem passar por diferentes tipos de tratamentos para potabilização. Atualmente, a eficiência de um sistema convencional completo (contando com as etapas de coagulação, floculação, decantação, filtração, desinfecção e polimento) largamente utilizado no Brasil ainda não foi completamente esclarecida. Nesse contexto, este trabalho teve o objetivo de avaliar o sistema de abastecimento público de Jaboticabal, através da avaliação da contaminação de $17 \beta$ estradiol e estrona no manancial superficial Córrego Rico.

\section{PARTE EXPERIMENTAL}

\section{Reagentes e padrões}

Foram utilizados padrões de estrogênios, com grau de pureza entre 100 e 99,4\%, para $17 \beta$ estradiol e estrona (Fluka). As soluçõesestoque foram preparadas em concentração de $10 \mathrm{mg} \mathrm{L}^{-1}$ para $17 \beta$ estradiol e $100 \mathrm{mg} \mathrm{L}^{-1}$ para estrona em acetonitrila, sendo armazenadas a $4{ }^{\circ} \mathrm{C}$ e ao abrigo da luz. Os solventes utilizados foram metanol (grau HPLC) e acetonitrila (grau HPLC) da J.T.Baker, hexano e acetona (grau HPLC) da Mallinckrodt.

\section{Preparo das amostras}

O preparo das amostras foi feito com extração em fase sólida, em cartuchos C18, com 500 mg e 6 mL (Accubond, Agilent-HP). Para todas as operações envolvendo extração em fase sólida, foi utilizado o conjunto de filtração (Millipore), operado em vazão de $6 \mathrm{~mL} \mathrm{~min}{ }^{-1}$. 
O condicionamento do cartucho foi feito com $6 \mathrm{~mL}$ de hexano, $2 \mathrm{~mL}$ de acetona, $6 \mathrm{~mL}$ de metanol e $10 \mathrm{~mL}$ de água $\mathrm{pH} 3 .{ }^{9}$

Após a passagem de $1 \mathrm{~L}$ de amostra pelo cartucho, previamente filtrada com filtros de $47 \mathrm{~mm}$ de diâmetro, $1,2 \mathrm{~mm}$ de diâmetro de poro (GF/C, Schleicher \& Schuell), foi feito o clean up do cartucho com $10 \mathrm{~mL}$ de $\mathrm{H}_{2} \mathrm{O}: \mathrm{MeOH}(9: 1$, v/v) e a secagem durante 1 h, sob vácuo. A eluição com $4 \mathrm{~mL}$ de acetona ocorreu por gravidade. $\mathrm{O}$ extrato em acetona foi evaporado até a secura e ressuspendido em $600 \mu \mathrm{L}$ de acetonitrila, sendo, em seguida, processado em filtro com $13 \mathrm{~mm}$ de diâmetro e 0,45 $\mu \mathrm{m}$ de diâmetro de poro (HV Millex, Durapore).

\section{Análise das amostras}

Foi utilizado cromatógrafo a líquido (Pro Star, com detector de fluorescência, modelo 360, e injetor automático modelo 400, acoplado a uma workstation Varian); coluna cromatográfica C18 (PAH, Supelco), com $250 \mathrm{~mm}$ de comprimento, 4,6 mm d.i., $5 \mu \mathrm{m}$ de tamanho de partícula e pré-coluna C18 (Phenomenex), com 30 mm de comprimento, 4,6 $\mathrm{mm}$ d.i. e $5 \mu \mathrm{m}$ de tamanho de partícula. Para a separação, foram utilizadas as seguintes condições: volume de injeção de $20 \mu \mathrm{L}$, vazão de $1,0 \mathrm{~mL} \mathrm{~min}{ }^{-1}$, eluição isocrática $\mathrm{H}_{2} \mathrm{O}: \mathrm{ACN}(1: 1, \mathrm{v} / \mathrm{v})$. A detecção foi feita em $230 \mathrm{~nm}$ (excitação) e $306 \mathrm{~nm}$ (emissão). ${ }^{26}$ A quantificação foi realizada pelo método do padrão externo, com curva analítica contendo cinco pontos, em concentrações de 0,013 a $0,100 \mathrm{mg} \mathrm{L}^{-1}$ para $17 \beta$ estradiol e de $1 \mathrm{a}$ $10 \mathrm{mg} \mathrm{L}^{-1}$ para estrona.

A validação do método utilizado foi publicada por Lopes et al. ${ }^{27}$ e conduzida em duas etapas. Primeiramente foram determinados parâmetros relacionados ao desempenho do cromatógrafo a líquido com detecção por fluorescência utilizando soluções-padrão (Tabela 1).

Tabela 1. Desempenho do cromatógrafo a líquido com detecção por fluorescência determinado experimentalmente para os estrogênios

\begin{tabular}{lcc}
\hline \multirow{2}{*}{ Parâmetros } & \multicolumn{2}{c}{ Estrogênios } \\
& Estrona & $17 \beta$ Estradiol \\
\hline $\begin{array}{l}\text { Intervalo de aplicação do } \\
\text { método (mg L }\end{array}$ & 1 a 10 & 0,013 a 0,100 \\
$\begin{array}{l}\text { Equações da curva analítica } \\
\text { Coeficiente de correlação }\left(\mathrm{r}^{2}\right)\end{array}$ & 0,997 & $\mathrm{Y}=1 \mathrm{x} 10^{7} \mathrm{x}+8424$ \\
$\begin{array}{l}\text { Coeficiente de variação do } \\
\text { fator de resposta (\%) }\end{array}$ & 3 & 0,999 \\
$\begin{array}{l}\text { Limite de detecção (mg L } \\
\text { Limite de quantificação }\end{array}$ & 0,5 & 4 \\
$\left(\mathrm{mg} \mathrm{L}^{-1}\right)$ & 1,0 & 0,006 \\
\hline
\end{tabular}

Na segunda etapa da validação, o procedimento analítico completo (extração e análise instrumental) foi submetido a ensaio de recuperação, em triplicata para três concentrações, com fortificação de amostra de água (1 L) pela adição de solução-padrão. Este foi repetido separadamente para água superficial e tratada devido à possibilidade da presença de diferentes interferentes (Tabela 2).

Os limites de detecção do método (LD) determinados foram de $3,7 \mathrm{ng} \mathrm{L}^{-1}$ para $17 \beta$ estradiol e de $300 \mathrm{ng} \mathrm{L}^{-1}$ para estrona, nas amostras de água superficial e tratada. ${ }^{27}$ Para tanto, o LD foi atribuído ao nível de fortificação da amostra em que havia identificação do pico da substância com área não-reprodutível em injeções sucessivas.

Os limites de quantificação do método (LQ) determinados para água superficial e tratada foram de 7,5 $\mathrm{ng} \mathrm{L}^{-1}$ para $17 \beta$ estradiol e de $600 \mathrm{ng} \mathrm{L}{ }^{-1}$ para a estrona. ${ }^{27}$ Para a determinação foi considerada a menor concentração de fortificação a apresentar área reprodutível em injeções sucessivas dentro do intervalo de trabalho, e com recuperação entre 40 e $120 \%$. A diferença entre os estrogênios pode ser
Tabela 2. Resultados da validação do método para exatidão e precisão para água superficial e tratada

\begin{tabular}{lcccccc}
\hline & \multicolumn{5}{c}{ Estrona } & \multicolumn{5}{c}{$17 \beta$ Estradiol } \\
Parâmetros & 600 & 1.500 & 5.000 & 7,5 & 30,0 & 50,0 \\
\hline Água superficial & & & & & & \\
Exatidão (Recuperação, \%) & 85 & 99 & 89 & 128 & 93 & 96 \\
Precisão (CV, \%)* & 18 & 3 & 10 & 8 & 3 & 13 \\
Água tratada & & & & & & \\
Exatidão (Recuperação, \%) & 85 & 83 & 92 & 86 & 93 & 86 \\
Precisão (CV, \%) & 6 & 5 & 5 & 24 & 9 & 5 \\
\hline
\end{tabular}

* Coeficiente de Variação.

atribuída à sensibilidade do detector à estrona, que apresentou menor fluorescência do que $17 \beta$ estradiol.

\section{Descrição da área e amostragem}

A área de desenvolvimento deste trabalho está inserida na Bacia Hidrográfica do Rio Mogi Guaçu, constituindo-se em parte da subbacia hidrográfica do Córrego Rico. O Córrego Rico possui vazão de 0,3 a 2,8 $\mathrm{m}^{3} \mathrm{~s}^{-1}$ no ponto de captação de água. ${ }^{28}$ Os pontos de coleta estavam localizados entre a nascente do Córrego Rico (CR) e a captação de água para abastecimento de Jaboticabal (CR1 - nascente; CR2 - após passagem pela área urbana do município de Monte Alto, distante $8,8 \mathrm{~km}$ da nascente; CR3 - no ponto de captação de água para abastecimento no município de Jaboticabal e distante $26,1 \mathrm{~km}$ da nascente, e PT4 - após passagem pela estação de tratamento de água). ${ }^{29} \mathrm{O}$ ponto $\mathrm{PT} 4$ refere-se à amostra de água da saída da ETA, com adição de cal, cloro, ortopolifosfato de sódio e ácido fluorsilícico, exceto para a coleta de agosto/2006, onde foi coletada água filtrada sem adição de produtos químicos.

$\mathrm{Na}$ área compreendida entre a divisa de Jaboticabal-Monte Alto e a captação de água em Jaboticabal, existem 92 propriedades rurais. ${ }^{30}$ Dessas, 33\% não produzem esgoto e as demais têm seus esgotos lançados em fossa séptica (56\%), a céu aberto (7\%) e nos córregos (4\%).

As colheitas foram feitas em período de cheia (março, outubro e novembro/2006) e de seca (agosto e setembro/2006). Nestas datas, as colheitas foram realizadas em horários fixos para cada ponto, para impedir a interferência da variação horária da composição do esgoto lançado no manancial na comparação entre períodos (seca e chuva).

Para complementar as avaliações, foram realizadas análises de turbidez, cor aparente e coliformes termotolerantes (CT), ${ }^{31}$ expressos em Unidade Nefelométrica de Turbidez (NTU), unidade Hazen (uH) e em Número Mais Provável (NMP) $100 \mathrm{~mL}^{-1}$, respectivamente. Realizou-se, também, análise de carbono orgânico dissolvido (COD), ${ }^{32}$ com filtração prévia em filtro com diâmetro de poro de $0,45 \mu \mathrm{m}$ e 13 mm de diâmetro do filtro (HV Millex, Durapore).

\section{RESULTADOS E DISCUSSÃO}

Os resultados obtidos para as coletas do período de cheia (março, outubro e novembro) e do período de seca (agosto e setembro), de 2006, estão expressos na Tabela 3. Os resultados para $17 \beta$ estradiol não foram expressos para as amostras de março/2006, pois o método utilizado não apresentava seletividade satisfatória na região de eluição desse hormônio.

O $17 \beta$ estradiol esteve presente em concentração quantificável (16,0-30,6 ng L $\mathrm{L}^{-1}$ ) no ponto CR1, uma nascente do Córrego Rico, nas amostras coletadas nos meses de agosto e setembro/2006, período de seca (Tabela 1). Neste caso, o relevo fortemente ondulado da região 
Tabela 3. Resultados de parâmetros indicadores de qualidade de água e concentração de estrona e $17 \beta$ estradiol nas coletas efetuadas em 2006 , na sub-bacia hidrográfica do Córrego Rico, em Jaboticabal e Monte Alto - SP

\begin{tabular}{|c|c|c|c|c|c|c|}
\hline Data & $\begin{array}{c}\text { Cor aparente } \\
(\mathrm{uH})\end{array}$ & $\begin{array}{c}\text { Turbidez } \\
\text { (uT) }\end{array}$ & $\begin{array}{c}\mathrm{COD} \\
\left(\mathrm{mg} \mathrm{L}^{-1}\right)\end{array}$ & $\begin{array}{c}\mathrm{CT} \\
\left(\mathrm{NMP} 100 \mathrm{~mL}^{-1}\right) \\
\end{array}$ & $\begin{array}{c}17 \beta \text { estradiol } \\
\left(\mathrm{ng} \mathrm{L}^{-1}\right)\end{array}$ & $\begin{array}{l}\text { Estrona } \\
\left.(\mathrm{ng} \mathrm{L})^{-1}\right) \\
\end{array}$ \\
\hline \multicolumn{7}{|l|}{$\overline{\mathrm{CR} 1}$} \\
\hline 24-03 & $<1$ & 0,2 & 3,61 & 86 & - & ND \\
\hline $27-03$ & $<1$ & 0,2 & 4,87 & $<1$ & - & ND \\
\hline 29-08 & 3 & 0,1 & - & $<1$ & 16,0 & ND \\
\hline 13-09 & $<1$ & 0,1 & $<1,22$ & $<1$ & ND & ND \\
\hline 28-09 & $<1$ & 0,2 & $<1,22$ & 18 & 30,6 & NQ \\
\hline $16-10$ & $<1$ & 0,2 & 2,50 & $<1$ & ND & ND \\
\hline $30-10$ & $<1$ & 0,2 & 4,58 & $<1$ & ND & ND \\
\hline 16-11 & $<1$ & 0,1 & 1,23 & $<1$ & ND & ND \\
\hline $22-11$ & $<1$ & 0,5 & 2,26 & $<1$ & ND & ND \\
\hline \multicolumn{7}{|l|}{ CR2 } \\
\hline $24-03$ & 381 & 52,1 & 7,30 & 2.500 & - & 600 \\
\hline $27-03$ & 185 & 24,8 & 5,67 & 900 & - & ND \\
\hline 29-08 & 81 & 7,6 & - & 468 & ND & ND \\
\hline 13-09 & 75 & 7,1 & 3,39 & 70 & ND & ND \\
\hline 28-09 & 208 & 14,5 & 6,56 & 1.700 & ND & ND \\
\hline $16-10$ & 165 & 21,7 & 5,65 & 310 & ND & ND \\
\hline $30-10$ & 214 & 25,9 & 11,70 & 183 & ND & ND \\
\hline 16-11 & 154 & 16,8 & 5,18 & 92 & 10,2 & ND \\
\hline $22-11$ & 143 & 15,8 & 6,51 & 184 & 8,6 & ND \\
\hline \multicolumn{7}{|l|}{ CR3 } \\
\hline 24-03 & 485 & 71,0 & 7,61 & 3.500 & - & ND \\
\hline 27-03 & 225 & 29,1 & 5,74 & 2.000 & - & ND \\
\hline 29-08 & 71 & 8,4 & - & 900 & ND & ND \\
\hline 13-09 & 57 & 6,2 & 1,93 & 120 & ND & ND \\
\hline 28-09 & 72 & 9,6 & 1,98 & 180 & 25,8 & ND \\
\hline $16-10$ & 103 & 15,6 & 6,55 & 200 & ND & ND \\
\hline $30-10$ & 155 & 20,6 & 7,58 & 265 & ND & ND \\
\hline 16-11 & 164 & 24,8 & - & 120 & ND & ND \\
\hline $22-11$ & 188 & 30,5 & 4,83 & 173 & ND & ND \\
\hline \multicolumn{7}{|l|}{ PT4 } \\
\hline 24-03 & $<1$ & 0,2 & $<1,22$ & 4 & - & ND \\
\hline 27-03 & $<1$ & 0,5 & 2,02 & 1 & - & ND \\
\hline 29-08 & 1 & 0,1 & - & 2 & ND & ND \\
\hline 13-09 & $<1$ & 0,1 & $<1,22$ & $<1$ & ND & ND \\
\hline 28-09 & $<1$ & 0,3 & $<1,22$ & $<1$ & 6,8 & ND \\
\hline $16-10$ & $<1$ & 0,3 & 3,82 & $<1$ & ND & ND \\
\hline $30-10$ & $<1$ & 0,1 & 6,48 & $<1$ & ND & ND \\
\hline 16-11 & $<1$ & 0,2 & 1,31 & $<1$ & ND & ND \\
\hline 22-11 & 1 & 0,3 & 2,86 & $<1$ & ND & ND \\
\hline
\end{tabular}

LQ: limite de quantificação (600 ng L ${ }^{-1}$ para Estrona e 7,5 ng L L para 17 estradiol). NMP: número mais provável. -: não analisado. ND: não detectado. NQ: não quantificado. uH: unidade Hazen. uT: unidade de turbidez. CT: coliformes trmotolerantes; COD: Carbono orgânico dissolvido.

é impróprio para a agricultura mecanizada e, historicamente, a área foi explorada com criação de gado e horticultura, o que demonstra a contribuição das atividades agrícolas para a dispersão de estrogênios no ambiente. Os resultados encontrados concordam com as concentrações apresentadas em nascentes ( 6 a $66 \mathrm{ng} \mathrm{L}^{-1}$ ) de região de criação de gado no Arkansas, conforme Peterson et al.. ${ }^{33}$

A estrona esteve presente na amostra coletada no período chuvoso, no ponto CR2. A amostra apresentou maior turbidez, cor aparente e CT. Esse fato pode ser atribuído ao escoamento superficial de áreas de criação animal. A presença de estrona em amostra de água superficial, na concentração de $600 \mathrm{ng} \mathrm{L}^{-1}$, esteve acima do valor observado em amostras para a Holanda, onde a estrona foi encontrada na concentração de $0,3 \mathrm{ng} \mathrm{L}^{-1}$ por Belfroid et al.. ${ }^{13}$ No entanto, esse valor está abaixo da concentração encontrada em corpos d'água do município de Campinas, onde esteve entre 3.500 e $5.000 \mathrm{ng} \mathrm{L}^{-1}{ }^{34}$ Rodriguez-Mozaz et al. encontraram concentrações de estrona de $22 \mathrm{ng} \mathrm{L}^{-1}$ no Rio Llobregat, Espanha, em período de seca, e de $<2,5$ a $8 \mathrm{ng} \mathrm{L}^{-1}$ em período de chuva. ${ }^{15}$ 
Tabela 4. Concentrações de estrogênios (ng L ${ }^{-1}$ ) obtidas neste estudo para o sistema de abastecimento de Jaboticabal - SP, e em estudos descritos na literatura

\begin{tabular}{|c|c|c|c|c|c|c|}
\hline \multirow{3}{*}{$\begin{array}{l}\text { Ponto de coleta } \\
\text { Nascente }\end{array}$} & \multicolumn{3}{|c|}{$17 \beta$ Estradiol } & \multicolumn{3}{|c|}{ Estrona } \\
\hline & \multirow{2}{*}{$\begin{array}{r}\text { Este estudo } \\
16,0-30,6\end{array}$} & \multicolumn{2}{|c|}{ Outros estudos } & \multirow{2}{*}{$\begin{array}{c}\text { Este estudo } \\
<600\end{array}$} & \multicolumn{2}{|c|}{ Outros estudos } \\
\hline & & $6-66$ & EUA $^{33}$ & & & \\
\hline \multirow{5}{*}{ Água superficial } & \multirow{5}{*}{$8,6-25,8$} & $1.900-6.000$ & Brasil $^{34^{*}}$ & 600 & $3.500-5.000$ & Brasil ${ }^{34^{*}}$ \\
\hline & & $<0,3-5,5$ & Holanda ${ }^{13}$ & & $<0,1-3,4$ & Holanda ${ }^{13}$ \\
\hline & & N. D. ${ }^{a}$ & EUA $^{16}$ & & N. D. ${ }^{a}$ & EUA $^{16}$ \\
\hline & & $0,15-3,6$ & Alemanha ${ }^{14}$ & & $0,10-4,1$ & Alemanha ${ }^{14}$ \\
\hline & & $38-2.510$ & Brasil ${ }^{35^{*}}$ & & $<2,5-22$ & Espanha $^{15}$ \\
\hline \multirow{3}{*}{ Água tratada } & \multirow{3}{*}{6,8} & $2.100-2.600$ & Brasil $^{34^{*}}$ & ND & N.D $D^{b}$ & Brasil ${ }^{34 *}$ \\
\hline & & N.D. ${ }^{a}$ & EUA $^{16}$ & & N. D. ${ }^{a}$ & EUA $^{16}$ \\
\hline & & $0,2-2,1$ & Alemanha $^{14}$ & & $0,20-0,60$ & Alemanha ${ }^{14}$ \\
\hline
\end{tabular}

* Campinas - SP; ND: não detectado. ${ }^{a}$ Limite de Detecção: estrona, $0,3 \mathrm{ng} \mathrm{L}{ }^{-1}, 17 \beta$ estradiol, $0,1 \mathrm{ng} \mathrm{L}^{-1}$. ${ }^{\mathrm{b}}$ Limite de Detecção: variou entre 2.400 e 3.300 ng $\mathrm{L}^{-1}$, em diferentes períodos de colheita.

Nas amostras coletadas em novembro de 2006 , observou-se a presença de $17 \beta$ estradiol no ponto CR2, entre 8,6 e 10,2 $\mathrm{ng} \mathrm{L}^{-1}$ (Tabela 1 ). Para o ponto CR3, observou-se a presença de $17 \beta$ estradiol em apenas uma das amostras do período de seca $\left(25,8 \mathrm{ng} \mathrm{L}^{-1}\right)$. Os resultados para turbidez destas amostras foram de 15,8; 16,8 e 9,6 NTU, respectivamente, e são característicos de ausência de chuva, o que contribui para a suspeita de que a redução de vazão do córrego leva à concentração de lançamentos de esgoto. As concentrações encontradas (8,6; 10,2 e $\left.25,8 \mathrm{ng} \mathrm{L}^{-1}\right)$ estão próximas à concentração mínima a apresentar efeito

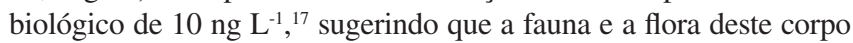
d'água estão suscetíveis a alterações das funções endócrinas.

Os resultados encontrados neste estudo estão abaixo das concentrações encontradas em Campinas - SP, por Ghiselli (1.900 a $6.000 \mathrm{ng}$ $\left.\mathrm{L}^{-1}\right) .{ }^{34}$ Sodré et al. também encontraram $17 \beta$ estradiol no Rio Atibaia, Campinas - SP, em níveis que estiveram acima do presente estudo (38 e $\left.2.510 \mathrm{ng} \mathrm{L}^{-1}\right) .{ }^{35}$ No último, as maiores concentrações foram encontradas em período de seca, reforçando a hipótese de lançamento de fontes pontuais como, por exemplo, esgoto doméstico. Para o Rio Jordão, as maiores concentrações também estiveram presentes no período de seca. ${ }^{17}$ Nesses casos, provavelmente, a evaporação colaborou com a redução da vazão do mesmo e concentrou os lançamentos de fontes pontuais. No entanto, a presença descontínua de estrogênios no manancial sugere que fontes difusas (além das citadas) contribuíram para o aporte de estrogênios para o manancial. Tal fato demonstra, também, que a variação do comportamento entre estações (chuva e seca) pode diferir nos anos seguintes, não só mediante as oscilações do regime hídrico, mas também de acordo com as alterações dos lançamentos dessas fontes difusas. Outros resultados descritos na literatura, para a presença de estrona e $17 \beta$ estradiol em águas superficiais, são mostrados na Tabela 4, e demonstram que as regiões brasileiras já pesquisadas apresentaram maiores concentrações de estrogênios.

A presença de $17 \beta$ estradiol na amostra do ponto CR3 coincidiu com a presença desse na água tratada de abastecimento público de Jaboticabal, em concentração de $6,8 \mathrm{ng} \mathrm{L}^{-1}$, sugerindo eficiência parcial $(73,3 \%)$ do processo de tratamento de água para a remoção dessa substância. De acordo com os conhecimentos atuais, o resultado para água tratada não infere risco à saúde humana, pois está abaixo da concentração encontrada como limiar para efeito biológico, ${ }^{17}$ no entanto, demonstra a necessidade de monitoramento frequente, pois sugere que, em algum momento, este limite pode ser ultrapassado. Rodriguez-Mozaz et al. não encontraram estrogênios em amostras de água da saída da estação de tratamento de Sant Join Despi, Espanha, contendo as etapas de pré-cloração, pré-dioxicloração, filtração em areia, ozonização e filtração em carvão ativado granular. ${ }^{15}$

Os resultados (Tabela 3) não mostraram clara correlação entre a concentração dos estrogênios e os parâmetros controlados pela legislação para águas naturais, expressos na Resolução Conama $357 / 05,{ }^{36}$ tal como é o caso da nascente (CR1), classificada nessa Resolução como classe 1, e da água superficial (CR2 e CR3), classificada como classe 2 (Tabela 5). Por meio de comparação, notou-se que estrona esteve presente em águas com maior cor aparente, turbidez e CT (24-03), contrariamente ao ocorrido com $17 \beta$ estradiol, que foi encontrado em águas quando os valores destes parâmetros eram baixos (29-08; 28-09; 16-11; 22-11). Estes resultados discordam dos obtidos por Rodriguez-Mozaz et al., que encontraram maiores concentrações de estrona em amostras com baixa turbidez. ${ }^{15}$

Tabela 5. Valores máximos permitidos para os parâmetros de qualidade de água analisados em amostras do estudo de caso de Jaboticabal - SP, de acordo com legislação vigente

\begin{tabular}{lccc}
\hline \multirow{2}{*}{ Parâmetro } & \multicolumn{2}{c}{ Resolução Conama 357 ${ }^{36}$} & \multirow{2}{*}{ Portaria 518 ${ }^{37}$} \\
\hline Cor aparente (uH) & 40 & 75 & 15 \\
Turbidez (uT) & - & 100 & 1 \\
CT (NMP 100 $\left.\mathrm{mL}^{-1}\right)$ & $200^{*}$ & $1.000^{*}$ & Ausente \\
Carbono Orgânico & - & - & - \\
Dissolvido (mg L & Classe & \\
Aplicáveis às amostras & CR1 & CR2, CR3 & Ponto 4 \\
\hline
\end{tabular}

*Em $80 \%$ de pelo menos seis amostras coletadas no período de 1 ano com frequência bimestral. - Parâmetro não controlado. NMP: número mais provável.

O carbono orgânico dissolvido variou entre $<1,22$ a $11,7 \mathrm{mg} \mathrm{L}^{-1}$ para água do Córrego Rico (Tabela 3) e entre 4,9 e 43,7 mg L-1 para corpos d'água de Campinas ${ }^{34} \mathrm{e}$, em ambos os casos, os resultados de COD não demonstraram relação com os estrogênios.

Por meio da comparação das Tabelas 3 e 5, pode-se notar que as águas pesquisadas atenderam parcialmente aos padrões expressos na legislação vigente, descritos por meio dos Valores Máximos Permitidos (VMPs) para água superficial ${ }^{36}$ e para água tratada. ${ }^{37}$

A porcentagem de amostras fora dos padrões ainda pode apresentar variação horária ao longo de um único dia, como ocorreu, por exemplo, para CT em amostras do ponto CR3. O monitoramento realizado no período de $24 \mathrm{~h},{ }^{31}$ mostrou que a contagem mínima ocorre entre 0 e 6 h (madrugada), com média de 833 Unidades Formadoras de Colônias (UFC) $100 \mathrm{~mL}^{-1}$ e a máxima entre 18 e 24 h (noite), com média de 1.567 UFC $100 \mathrm{~mL}^{-1}$. Esta oscilação pode ser atribuída à variação da composição do esgoto doméstico lançado no corpo d'água, e a principal consequência consiste na necessidade de se ajustar o processo de tratamento às alterações da qualidade da água. 
No período de chuva, a cor aparente das amostras de água superficial (CR2 e CR3) esteve acima do limite $(75 \mathrm{uH})$ estabelecido na legislação, em valores muito maiores (103 a $485 \mathrm{uH})$ que o valor excedido para período de seca (71 a $208 \mathrm{uH})$. Esses dados concordam com os valores de turbidez, COD (que também foram maiores em período de cheia) e CT. No entanto, a ausência de correlação entre os estrogênios e os parâmetros indicadores de qualidade de água concorda com a suspeita da existência de diversas fontes difusas com lançamentos intermitentes. Destaca-se, ainda, que há uma dispersão de estrogênios entre amostras que atendem e que não atendem à legislação, indicando que o enquadramento legal não oferece segurança quanto a este grupo de contaminantes.

\section{CONCLUSÃO}

Os estrogênios estiveram presentes na água do Córrego Rico de forma descontínua e em nível passível de provocar alterações fisiológicas nos organismos aquáticos. Apesar disso, a presença de $17 \beta$ estradiol em água tratada esteve abaixo do nível passível de exercer efeito biológico. Para os pontos CR1, CR3 e PT4 os estrogênios foram quantificados em período de seca (agosto e setembro/2006) e, para o ponto CR2, em período de chuva (março e novembro/2006). Nos pontos CR1 e CR2, os resíduos animais e os lançamentos de esgoto de Monte Alto podem ter sido as maiores fontes de aporte de estrogênios para a água e no ponto CR3, os lançamentos de esgotos domésticos não tratados das propriedades. No entanto, a ausência de correlação entre a concentração de estrogênios e outros parâmetros indicadores de qualidade de água mostrou que outras fontes difusas ao longo do trecho estudado podem ter colaborado com o aporte de estrogênio para o manancial.

\section{AGRADECIMENTOS}

Ao Serviço Autônomo de Água e Esgoto de Jaboticabal, pelo apoio. À FAPESP, pelo apoio financeiro.

\section{REFERÊNCIAS}

1. Bila, D. M.; Dezotti, M.; Quim. Nova 2003, 26, 523.

2. Bila, D. M.; Dezotti, M.; Quim. Nova 2007, 30, 651; Litelmann, J.; Katayama, A.; Kurihara, N.; Shore, L.; Wenzel, A.; Pure Appl. Chem. 2003, 75, 631; Reis Filho, R. W.; Araújo, J. C.; Vieira, E. M.; Quim. Nova 2006, 29, 817; Ghiselli, G.; Jardim, W. F.; Quim. Nova 2007, 30, 695; http://lqa.iqm.unicamp.br acessado em Fevereiro 2010.

3. Fieser, L. F.; Fieser, M.; Steroids, VNR: New York, 1959.

4. Fent, K. Em Endocrinically active chemicals in the environment; Gies, A., org.; Federal Environmental Agency: Berlim, 1996, p. 69-81.

5. Jobling, S.; Nolan, M.; Tyler, C. R.; Brighty, G.; Sumpter, J. P.; Environ. Sci. Technol. 1998, 32, 2498.

6. Daston, G. P.; Gooch, J. W.; Breslin, W. J.; Sherey, D. L.; Nikiforov, A. I.; Fico, T. A.; Gorsuch, J. W.; Reproductive Toxicology 1997, 11, 465.

7. Sharpe, R. M.; Skakkebaek, N. E.; Lancet 1993, 341, 1392.

8. Halling-Sorensen, B.; Nielsen, S. N.; Lanzky, P. F.; Ingerslev, F.; Lutzhoft, H. C. H.; Jorgensen, S. E.; Chemosphere 1998, 36, 357.
9. Lee, H.-B.; Peart, T. E.; J. AOAC Int. 1998, 81, 1209.

10. Ternes, T. A.; Stumpf, M.; Mueller, J.; Haberer, K.; Wilken, R.-D.; Servos, M.; Sci. Total Environ. 1999, 225, 81.

11. Baronti, C.; Curini, R.; D’Ascenzo, G.; Di Corsia, A.; Gentili, A.; Samperi, R.; Environ. Sci. Technol. 2000, 34, 5049.

12. Araújo, J. C.; Dissertação de Mestrado, Universidade de São Paulo, Brasil, 2006.

13. Belfroid, A. C.; van der Horst, A.; Vethaak, A. D.; Schäfer, A. J.; Rijis, G. B. J.; Wegener, J.; Confino, W. P.; Sci. Total. Environ. 1999, 225, 101.

14. Kuch, H. M.; Ballschmiter, K.; Environ. Sci. Technol. 2001, 35, 3201.

15. Rodriguez-Mozaz, S.; Alda, M. J. T.; Barceló, D.; J. Chromatogr., A 2004, 1045, 85 .

16. Boyd, G. R.; Reemtsma, H.; Grimm, D. A.; Mitra, S.; Sci. Total Environ. 2003, 311, 135 .

17. Barel-Cohen, K.; Shore, L. S.; Shemesh, M.; Wenzel, A.; Mueller, J.; Kronfeld-Schor, N.; J. Environ. Manag. 2006, 78, 16.

18. Desbrow, C.; Routledge, E. J.; Brighty, G. C.; Sumpter, J. P.; Waldock, M.; Environ. Sci. Technol. 1998, 32, 1549.

19. Aherne, G. W.; Briggs, R.; J. Pharm. Pharmacol. 1989, 41, 735.

20. D’Ascenzo, G.; Di Corcia, A.; Gentili, A.; Mancini, R.; Mastropasqua, R.; Nazzari, M.; Samperi, R.; Sci. Total Environ. 2003, 302, 199.

21. Lee, H. B.; Liu, D.; Water, Air, Soil Pollut. 2002, 134, 351.

22. Holthaus, K. I. E.; Johnson, S. C.; Jurgens, M. D.; Williams, R. J.; Smith, J. J. L.; Carter, J. E.; Environ. Toxicol. Chem. 2002, 21, 2526.

23. Lai, K. M.; Johnson, K. L.; Scrimshaw, M. D.; Lester, J. N.; Environ. Sci. Technol. 2000, 34, 3890.

24. Lee, L. S.; Strock, T. J.; Sarmah, A. K.; Suresh, P.; Rao, C.; Sci. Environ. Technol. 2003, 37, 4098.

25. Jürgens, M. D.; Holthaus, K. I. E.; Johnson, A. C.; Smith, J. J. L.; Hetheridge, M.; Williams, R. J.; Environ. Toxicol. Chem. 2002, 21, 480.

26. Snyder, S. A.; Keith, T. L.; Verbrugge, D. A.; Snyder, E. M.; Gross, T. S.; Kannan, K.; Giesy, J. P.; Environ. Sci. Technol. 1999, 33, 2814.

27. Lopes, L. G.; Marchi, M. R. R.; Souza, J. B. G.; Moura, J. A.; Cruz, C.; Analytica 2008, 37, 56.

28. Lopes, L. G.; Italiano, W. L.; Costa Jr., L. L.; Hojaij, A.; Galbiatti, J. A.; Marques Jr., S. A.; Anais do XXXII Congresso Brasileiro de Engenharia Agrícola, Goiânia, Brasil, 2003.

29. Zanini, H. L. H. T.; Tese de Doutorado, Universidade Estadual Paulista, Brasil, 2009.

30. Giacometti, L.; Costa Jr., L. L.; Gestão hídrica no Município de Jaboticabal: Bacia Hidrográfica do Córrego Rico, SAAEJ: Jaboticabal, 2002.

31. Apha; Standard methods for the examination of water and wastewater, American Public Health Association: Washington, 1998.

32. Martini, R. A.; Dissertação de Mestrado, Universidade Estadual Paulista, Brasil, 2005.

33. Peterson, E. W.; Davis, R. K.; Orndorff, H. A.; J. Environ. Qual. 2000, $29,826$.

34. Ghiselli, G.; Tese de Doutorado, Universidade Estadual de Campinas, Brasil, 2006.

35. Sodré, F. F.; Montagner, C. C.; Locatelli, M. A. F.; Jardim, W. F.; J. Bras. Soc. Ecotoxicol. 2007, 2, 187.

36. Brasil. Ministério do Meio Ambiente. Conselho Nacional do Meio Ambiente; Resolução n. 357, de 17 de março de 2005.

37. Brasil. Ministério da Saúde; Portaria n. 518, de 25 de março de 2004. 